\title{
SOME COMMUTATIVITY RESULTS FOR RINGS
}

\author{
Abraham A. Klein, Itzhak Nada, \\ AND HOWARD E. BELL
}

It is proved that certain rings satisfying generalized-commutator constraints of the form $\left[x^{m}, y^{n}, y^{n}, \ldots, y^{n}\right]=0$ must have nil commutator ideal.

Let $R$ be an associative ring; and define generalized commutators $\left[x_{1}, x_{2}, \ldots, x_{k}\right], k \geq 2$, as follows: $\left[x_{1}, x_{2}\right]=x_{1} x_{2}-x_{2} x_{1} ;$ and for $k>2,\left[x_{1}, x_{2}, \ldots, x_{k}\right]=\left[\left[x_{1}, \ldots, x_{k-1}\right], x_{k}\right]$. For $x_{1}=x$ and $x_{2}=x_{3}=\ldots=x_{k}=y$, abbreviate $[x, y, \ldots, y]$ by $[x, y]_{k}$.

A few years ago it was proved independently by Herstein [2] and by Anan' in and Zyabko [1] that $R$ has nil commutator ideal if for each $x_{1}, x_{2} \in R$ there exist positive integers $n_{1}=n_{1}\left(x_{1}, x_{2}\right)$ and $n_{2}=n_{2}\left(x_{1}, x_{2}\right)$ such that $\left[x_{1}^{n_{1}}, x_{2}^{n_{2}}\right]=0$; more recently Herste in [3] has established the same conclusion under the hypothesis that for all $x_{1}, x_{2}, x_{3} \in R$ there are positive integers $n_{1}, n_{2}, n_{3}$ such that $\left[x_{1}^{n}, x_{2}^{n_{2}}, x_{3}^{n_{3}}\right]=0$. The following conjecture arises naturally from this work.

CONJECTURE. Let $k>1$ and suppose that for each $x, y \in R$, there

Received 17 April 1980. Professor Howard E. Bell was supported by the Natural Sciences and Engineering Research Council of Canada, Grant No. A3961. 
exist positive integers $m, n$ such that $\left[x^{m}, y^{n}\right]_{k}=0$. Then the commutator ideal of $R$ is nil.

Given the complexity of [2] and [3], it would appear that no proof of this conjecture is in sight; indeed, even the $k=3$ case seems difficult. Hence the following case may be of interest.

THEOREM 1. Let $R$ be a ring and let $M$ be a fixed positive integer. Suppose that for each $x, y \in R$ there exist positive integers $m=m(x, y) \leq M$ and $n=n(x, y)$ such that $\left[x^{m}, y^{n}, y^{n}\right]=0$. Then the commitator ideal of $R$ is niz.

Proof. By proceeding as in [3], we can reduce the problem to establishing commutativity of $R$ under the additional hypotheses that $R$ is prime and torsion-free, and that every element of $R$ is either regular or nilpotent - hypotheses which we henceforth assume. Moreover, in view of the result of [1] and [2], we need only show that for each $x, y \in R$, there exist $m, n$ for which $\left[x^{m}, y^{n}\right]=0$.

Clearly this condition holds for nilpotent $y$, so we assume that $y$ is regular, and choose $m \leq M$ and $n_{1}$ for which $\left[x^{m}, y^{n_{1}}, y^{n_{1}}\right]=0$. Taking $x_{1}=x^{2 m}$, let $w$ and $n_{2}$ be such that $\left[x_{1}^{w}, y^{n_{2}}, y^{n_{2}}\right]=0$; and note that for $v=a w$ and $n=n_{1} n_{2}$, we have $\left[x^{m}, y^{n}, y^{n}\right]$ and $\left[x^{m v}, y^{n}, y^{n}\right]=0$, so that $\left[x^{m}, y^{n}\right]$ is nilpotent by $[3$, Lemma 1$]$. Thus, if $a$ is chosen to be an appropriate power of $\left[x^{m}, y^{n}\right]$ and $z=y^{n}$, we nave $a^{2}=[a, z]=0$.

For any $u \in R$ and $i \geq 1$, there exist $m_{i} \leq M$ and $s_{i}$ such that $\left[u^{m},(i z+a)^{s} i,(i z+a)^{s} i\right]=0$. Taking $i=1,2, \ldots, 2 M+1$ and using the pigeon-hole principle, we get $i_{1}, i_{2}, i_{3}$ with $1 \leq i_{1}<i_{2}<i_{3} \leq 2 M+1$ for which $m_{i_{1}}=m_{i_{2}}=m_{i_{3}}$. Denoting this common value by $q$ and defining $s=s_{i_{1}} s_{i_{2}} s_{i_{3}}$, we have $\left[u^{q},\left(i_{j} z+a\right)^{s},\left(i_{j}^{z+a}\right)^{s}\right]=0$, 
$j=1,2,3$; it now follows by use of the fact that $a^{2}=[a, z]=0$ that

$$
i_{j}^{2 s} v_{1}+i v_{j}^{2 s-1} v_{2}+i_{j}^{2 s-2} v_{3}=0, j=1,2,3,
$$

where $v_{1}, v_{2}, v_{3}$ are respectively defined to be $\left[u^{q}, z^{s}, z^{s}\right]$, $2 s\left[u^{q}, z^{s-1} a, z^{s}\right]$ and $s^{2}\left[u^{q}, z^{s-1} a, z^{s-1} a\right]$. The $3 \times 3$ coefficient matrix in ( $I$ ) is obtained by multiplying the rows of a Vandermonde matrix by non-zero integers, so the fact that $R$ is torsion-free yields $v_{1}=v_{2}=v_{3}=0$; and since $a^{2}=0=\left[a, z^{s-1}\right]$ and $z$ is regular, the statement $v_{3}=0$ reduces to the result that $a u_{a} a=0$.

If $b \in R$ and $b^{2}=0$, we claim that $a b a=0$. For if $v \in R$, there exists $q \leq M$ for which $a(a v a b+b)^{q} a=0$, which yields $a b(a v a b)^{q-1} a=0=(a b a v)^{q}$. Thus $a b a R$ is a nil right ideal of bounded index, which by the Nagata-Higman Theorem [4, p. 274] must be nilpotent; and the primeness of $R$ forces $a b a=0$.

Now if $c, d \in R$ with $c d=0,(d v c)^{2}=0$ for arbitrary $v \in R$, and hence advea $=0$. Since $R$ is prime, we have $a d=0$ or $c a=0$, so $c a d=0$. Thus, insertion of $\alpha$ as a factor preserves triviality of products; and from $a u^{q} a=0$ we can conclude $(a u)^{q+1}=0$. Therefore $(a u)^{M+1}=0$ for all $u \in R$, and another appeal to the Nagata-Higman Theorem gives $a=0$. Thus we have that any power of the nilpotent element $\left[x^{m}, y^{n}\right]$ whose square is 0 must also be 0 , so $\left[x^{m}, y^{n}\right]=0$. The proof of Theorem 1 is now complete.

The following theorem, except of having its own interest, shows that the conjecture is implied by the Köthe Conjecture.

THEOREM 2. Let $R$ be a ring with no non-zero nil right ideals, and $l \in t>1$. Suppose that for each $x, y \in R$ there exist $m, n \geq 1$ such that $\left[x^{m}, y^{n}\right]_{k}=0$. Then $R$ is commutative.

Proof. Let $a$ be in $R$ with $a^{2}=0$, and let $x$ be an arbitrary 
element of $R$. Take $m, n>1$ such that $\left[(a+a x)^{m},(a x)^{n}\right]_{k}=0$. This condition reduces to $(a x)^{t} a=0$ where $t=m-1+(k-1) n$, hence $a R$ is nil, so $a=0$. Consequently, $R^{2}$ has no non-zero nilpotent elements; and by a well-known result it is a subdirect product of domains. Our proof will be complete once we establish that each of these domains must be commutative. This is easily verified as in [3] for such a domain of prime characteristic; and such a torsion-free domain is commutative by the following lemma.

LEMMA. Let $R$ be a torsion-free domain, and let $k>1$. Suppose that for each $x, y \in R$, there exist $m, n \geq 1$ such that $\left[x^{m}, y^{n}\right]_{k}=0$. Then $R$ is commutative.

Proof. Assume $k \geq 3$ and let $x, y \in R$. Then there exist $m, r_{1}$ such that $\left[x^{m}, y^{r}\right]_{k}=0$ and there exist $m^{\prime}, r_{2}$ such that $\left[x^{(2 m) m^{\prime}}, y^{r}\right]_{k}=0$. It can easily be verified that

$$
\left[x^{m}, y^{r}\right]_{k}=\left[x^{(2 m) m^{\prime}}, y^{r}\right]_{k}=0
$$

for $r=r_{1} r_{2}$. Taking $x_{0}=x^{m}$ and $t=2 m^{\prime}$ and letting $\delta$ be the derivation defined by $u \delta=\left[u, y^{r}\right]$, we have $x_{0} \delta^{k-1}=\left(x_{0}^{t}\right) \delta^{k-1}=0$. Now $t \geq 2$ and $k \geq 3$, so $t(k-2) \geq k-1$ and therefore $\left(x_{0}^{t}\right) \delta^{t(k-2)}=0$. Expanding this last equation and using the fact that $x_{0} \delta^{k-1}=0$, we obtain a non-zero integer $s$ for which $s\left(x_{0} \delta^{k-2}\right)^{t}=0$ and our hypotheses on $R$ yield $x_{0} \delta^{k-2}=0$, which we may express as $\left[x^{m}, y^{r}\right]_{k-1}=0$. Thus we work back to the $k=2$ case of [1] and [2].

The entire problem becomes much more tractable for rings with 1 . Indeed, we can establish the following theorem.

THEOREM 3. For all $k>1$ the conjecture is true for rings $R$ with 1. 
We omit the details of the proof. The computational details are similar to the ones already presented. We merely note that it suffices to establish commutativity of $R$ under the additional hypotheses that $R$ is prime and torsion-free. A Vandermonde argument is used to prove that if these additional hypotheses hold, then $R$ has no non-zero nilpotent elements so it is a domain, and it is commutative by the result of the lemma.

\section{References}

[1] А.З. Ананьин, E.M. Знбно [A.Z. Anan'in, E.M. Zyabko], "Об одном вопросе Фейса" [On a question due to Faith], AZgebra i Logika 13 $(1974), 125-131$.

[2] I.N. Herstein, "A commutativity theorem", J. Algebra 38 (1976), $112-118$.

[3] I.N. Herstein, "On rings with a particular variable identity", $J$. Algebra 62 (1980), 346-357.

[4] Nathan Jacobson, Structure of rings (Amer. Math. Soc. Colloquium Publications, 37. American Mathematical Society, Providence, Rhode Island, 1956. Revised edition, 1964).

Dr Abraham A. Klein and $\mathrm{Dr} I$ tzhak Nada,

Department of Mathematical Sciences,

Tel-Aviv University,

Ramat-Aviv,

|srael;

Professor Howard E. Bell,

Department of Mathematics,

Brock University,

St Catharines,

Ontario,

Canada L2S 3AI. 